\title{
Gene Expression Profile of the A549 Human Non-Small Cell Lung Carcinoma Cell Line following Treatment with the Seeds of Descurainia sophia, a Potential Anticancer Drug
}

\author{
Bu-Yeo Kim, Jun Lee, Sung Joon Park, Ok-Sun Bang, and No Soo Kim \\ KM-Based Herbal Drug Research Group, Herbal Medicine Research Division, Korea Institute of Oriental Medicine, \\ Daejeon 305-811, Republic of Korea \\ Correspondence should be addressed to No Soo Kim; nosookim@kiom.re.kr
}

Received 3 April 2013; Revised 27 May 2013; Accepted 5 June 2013

Academic Editor: Bo-Hyoung Jang

Copyright (C) $2013 \mathrm{Bu}$-Yeo Kim et al. This is an open access article distributed under the Creative Commons Attribution License, which permits unrestricted use, distribution, and reproduction in any medium, provided the original work is properly cited.

\begin{abstract}
Descurainia sophia has been traditionally used in Korean medicine for treatment of diverse diseases and their symptoms, such as cough, asthma, and edema. Our previous results showed that ethanol extract of the seeds of D. sophia (EEDS) has a potent cytotoxic effect on human cancer cells. In this study, we reveal the molecular events that are induced by EEDS treatment in A549 human lung cancer cells. The dose-dependent effect of EEDS on gene expression was measured via a microarray analysis. Gene ontology and pathway analyses were performed to identify functional involvement of genes regulated by EEDS. From gene expression analyses, two major dose-dependent patterns were observed after EEDS treatment. One pattern consisted of 1,680 downregulated genes primarily involved in metabolic processes (FDR $<0.01$ ). The second pattern consisted of 1,673 upregulated genes primarily involved in signaling processes $($ FDR $<0.01)$. Pathway activity analyses revealed that the metabolism-related pathways and signaling-related pathways were regulated by the EEDS in dose-dependent and reciprocal manners. In conclusion, the identified biphasic regulatory mechanism involving activation of signaling pathways may provide molecular evidence to explain the inhibitory effect of EEDS on A549 cell growth
\end{abstract}

\section{Introduction}

Public health statistics indicate that neoplastic disease (commonly referred to as cancer) is a leading cause of death in the Republic of Korea, where more than 142 cancer-related deaths per 100,000 people occurred in 2011 (http://kostat.go.kr). Although a wide-range of anticancer drugs that target cancerrelated molecules have been developed, the five-year relative survival rate of cancer patients, especially those with lung cancer, has not improved significantly (http://www.cancerresearchuk.org/cancer-info/cancerstats/survival/commoncancers/). This disappointing clinical outcome may be a consequence of the multifactorial nature of cancer and the acquisition of drug resistance by tumor cells $[1,2]$. For these reasons, anticancer chemotherapy is now shifting from mono-substance therapy to combination therapy [3-5]. Extracts of medicinal herbs represent promising sources of novel multi-substance anticancer drugs [3].
Descurainia sophia (L.) Webb ex Prantl (Flixweed) is widely distributed in northeastern China and belongs to the family Brassicaceae (Cruciferae). In traditional Korean medicine (KM), the seeds of $D$. sophia have been used for the treatment of diverse diseases and their symptoms, such as cough, asthma, and edema [6]. According to the results of previous studies, $D$. sophia possesses biologically active secondary metabolites, such as cardiac glycosides [7], sulfur glycoside [8], nor-lignan [9], and lactones [10]. In our in vitro cytotoxic pre-screening system, the ethanol extract of D. sophia seeds (EEDS) displayed potent cytotoxicity against diverse human cancer cells. In addition, cytotoxic (helveticoside) and anti-inflammatory (quercetin and syringaresinol) active constituents were isolated from the EEDS [6].

Although the therapeutic constituents we identified in the EEDS have been well-characterized, the diverse composition of herbal extracts makes it difficult to elucidate their exact molecular mechanisms. Moreover, considering that a number 
of genes regulated by herbal extracts exert combined effects on various biological pathways, it is important to study the effects of herbal extracts at the genomic and molecular levels rather than at the individual gene level. Recent advances in the multi-target/multi-substance therapeutic approach have underscored the importance of using high-throughput analyses to identify the therapeutic mechanisms of complex drugs, such as herbal extracts [11]. Therefore, in the present study, we measured the in vitro anti-proliferative effects of the EEDS on human lung cancer cells and developed a gene expression profile using a microarray analysis. Dose-dependent analyses of the microarray data revealed that biological functions associated with signal transduction, such as apoptosis, were significantly elevated after EEDS treatment.

\section{Materials and Methods}

2.1. Plant Materials. The dried seeds of D. sophia were purchased from the Kwangmyungdang Medicinal Herbs Co. (Ulsan, Republic of Korea) and identified by Dr. Go Ya Choi, Basic Herbal Medicine Research Group, Herbal Medicine Research Division, Korea Institute of Oriental Medicine, Republic of Korea. A voucher specimen (KIOM-CRC-5) was deposited at the Cancer Research Center, Herbal Medicine Research Division, Korea Institute of Oriental Medicine, Republic of Korea.

2.2. Preparation of EEDS. The dried seeds $(9.0 \mathrm{~kg})$ of $D$. sophia were ground and extracted by maceration ( $40 \mathrm{~L}$ of $80 \%$ EtOH for $48 \mathrm{~h}, 3$ times) at room temperature. The combined extracts were filtered through Whatman filter paper (No. 2, Whatman International, Maidstone, UK) and concentrated using an EYELA rotary evaporation system (20 L, Tokyo Rikakikai, Tokyo, Japan) at $40^{\circ} \mathrm{C}$ to yield a two-phase extract $(766.1 \mathrm{~g})$, which consisted of an upper oil phase and a lower solid phase. The oil phase did not affect the proliferation of A549 cells. Therefore, we did not test the oil phase for further studies. The solid lower extract $(535.7 \mathrm{~g})$ was dried in a WiseVen vacuum oven (WOW-70, Daihan Scientific, Seoul, Republic of Korea) at $40^{\circ} \mathrm{C}$ for $24 \mathrm{~h}$ prior to use. The dried solid ethanol extract, that is EEDS, was dissolved in $100 \%$ dimethylsulfoxide (DMSO, Sigma, St Louis, MO, USA) at a concentration of $20 \mathrm{mg} / \mathrm{mL}$ and stored at $-80^{\circ} \mathrm{C}$.

2.3. Cell Lines and Culture Conditions. All human lung cancer cell lines, including the A549 cells, and the IMR-90 normal lung fibroblast cells used in this study were obtained from the American Type Culture Collection (ATCC, Manassas, VA, USA). All cells, with the exception of IMR-90 (DMEM), were grown in RPMI1640 (Invitrogen, Carlsbad, CA, USA) that had been supplemented with $10 \%(\mathrm{v} / \mathrm{v})$ fetal bovine serum (FBS) (Invitrogen), $100 \mathrm{U} / \mathrm{mL}$ of penicillin, and $100 \mu \mathrm{g} / \mathrm{mL}$ of streptomycin (Invitrogen) in $5 \% \mathrm{CO}_{2}$ humidified air at $37^{\circ} \mathrm{C}$.

2.4. Cell Proliferation Assays. Cell viability was quantified in a 96-well tissue culture plate using the Ez-Cytox cell proliferation assay kit (Daeil Lab Service, Seoul, Republic of Korea), as previously described [12]. Briefly, $5 \times 10^{3}$ cells were seeded on culture plates containing $100 \mu \mathrm{L} /$ well of culture medium. After $24 \mathrm{~h}$, the cells were exposed to various concentrations of the EEDS and maintained for the indicated time periods. The maximum concentration of DMSO vehicle was $0.5 \%(\mathrm{v} / \mathrm{v})$. Following drug treatment, the cells were washed with phosphate-buffered saline (PBS) to minimize the interference of the EEDS with the Ez-Cytox reaction. Color development in the Ez-Cytox solution by live cells was monitored at $450 \mathrm{~nm}$ using the Emax microplate reader (Molecular Devices, Sunnyvale, CA, USA).

2.5. Colony Forming Assays. A549 cells were seeded on 6well culture plates at a density of 200 cells/well and grown for $24 \mathrm{~h}$. The cells were exposed to various concentrations of the EEDS or the vehicle control for $72 \mathrm{~h}$. The culture medium was removed, and after a brief wash with PBS, the cells were grown for 10 days in fresh medium that did not contain the EEDS. After a brief wash with ice-cold PBS, the cells were fixed using an ice-cold neutralized $4 \%(\mathrm{w} / \mathrm{v})$ paraformaldehyde solution (Biosesang, Seongnam, Republic of Korea) for $10 \mathrm{~min}$. After removing the fixation solution, the colonies were stained with a $0.05 \%(\mathrm{w} / \mathrm{v})$ crystal violet (Sigma) cell staining solution for $30 \mathrm{~min}$. The free crystal violet solution was removed, and the cells were washed two times with tap water. The stained colonies were photographed, and the number of colonies was manually counted.

2.6. FACS Analysis. Apoptotic cell death was determined using the fluorescein isothiocyanate (FITC)-Annexin V apoptosis detection kit (BD Biosciences, San Jose, CA, USA) according to the manufacturer's instruction. In brief, A549 cells were seeded on 6-well culture plates at a density of $2 \times 10^{5}$ cells/well. After $24 \mathrm{~h}$, cells were exposed to EEDS ( 0 or $20 \mu \mathrm{g} / \mathrm{mL}$ ) for the indicated time periods. Cells were harvested, washed two times with ice-cold PBS, and resuspended in $100 \mu \mathrm{L}$ of $1 \mathrm{x}$ binding buffer. Then, $5 \mu \mathrm{L}$ of FITCAnnexin $\mathrm{V}$ and $5 \mu \mathrm{L}$ of propidium iodide (PI) solution were added to the cells, and the mixture was incubated at room temperature for $15 \mathrm{~min}$ in the dark. After addition of $400 \mu \mathrm{L}$ of $1 x$ binding buffer, the cells were analyzed by flow cytometry (FACSCalibur, BD Biosciences).

2.7. Microarray Experiment. Total RNA from A549 cells that had been treated with either the EEDS or the vehicle control was prepared using the Easy-Spin total RNA extraction kit (iNtRON Biotechnology, Seoul, Republic of Korea) in accordance with the manufacturer's instructions. Before performing the microarray experiment, the quality of the isolated total RNA was confirmed by electropherogram. RNA integrity number $(\mathrm{RIN})=9.8-10.0, \mathrm{OD}_{260 / 280}(>2.0)$, and $\mathrm{OD}_{260 / 230}(>2.2)$ were determined (see Supplementary Figure 1 and Supplementary Table 1 of the Supplementary Material online at http://dx.doi.org/10.1155/2013/584604), as previously described [13], by Genomictree, Inc. (Daejeon, Republic of Korea). Total RNA was amplified and labeled using the Low RNA Input Linear Amplification kit PLUS (Agilent Technologies, Santa Clara, CA, USA) and then hybridized to a microarray (Agilent Human whole genome 
$44 \mathrm{~K}$, Agilent Technologies) containing approximately 44,000 probes ( 21,600 unique genes), in accordance with the manufacturer's instructions. The arrays were scanned using an Agilent DNA Microarray Scanner.

\subsection{Semiquantitative PCR ( $q P C R)$. Single-stranded cDNA} was synthesized from $1 \mu \mathrm{g}$ of total RNA using the SuperScript III first-strand synthesis system (Invitrogen) according to the manufacturer's instruction. The concentration of cDNA was quantified using the ND-1000 NanoDrop spectrophotometer (Thermo Scientific, Wilmington, DE, USA), and $100 \mathrm{ng}$ of cDNA was used as a template for semi-qPCR reaction. The PCR products were analyzed by $1.5 \%$ agarose gel electrophoresis. The information of primer sequences and PCR reaction conditions are summarized in the Supplementary Table 3.

2.9. Dose-Dependent Microarray Analyses. The raw signal intensities were obtained using Agilent Feature Extraction Software (Agilent Technologies). Array elements with signal intensities below 1.4-fold of the local background were eliminated, and then the remaining elements were normalized using the quantile method [14]. After averaging the ratio of duplicated spots, the expression ratios were hierarchically clustered using the CLUSTER program (http://rana.lbl.gov/). The short time series expression miner (STEM) program, which was originally developed for the temporal analysis of gene expression [15], was used to identify dose-dependently expressed genes. The statistical significance of the resultant expression pattern was calculated as a false discovery rate (FDR) using 1,000 random permutations.

2.10. Public Microarray Dataset. The publically available microarray dataset with accession number of GSE4573, archived in the Gene Expression Omnibus (http://www.ncbi .nlm.nih.gov/geo), was used in the present study [16]. The dataset was composed of 130 squamous lung carcinoma tissues with survival information. We normalized the probe intensities of each array using the quantile method [14]. After averaging multiple probes, genes that were associated with survival were selected using BRB ArrayTools (version 4.2.1, http://linus.nci.nih.gov/BRB-ArrayTools.html), which compute a statistical significance for each gene using a Cox proportional hazard regression model with a univariate permutation test number of 10,000 .

2.11. Gene Ontology (GO) Analyses. The Functional Annotation Tool of DAVID [17] and the High-Throughput GoMiner algorithm [18] were used for simple and dose-dependent GO analyses, respectively. Only the list of genes was used for DAVID, while both the list of genes and the expression ratios were applied as inputs for the GoMiner. In both cases, the $P$ value of each GO-term was calculated using Fisher's exact test. For adjustments of multiple comparisons, the Benjamini-Hochberg procedure was used for DAVID [17]. For the GoMiner analysis, a random sampling-based FDR was calculated from 1,000 iterations. The resultant significant GO-terms from the GO analysis were entered into the REVIGO program to construct a network structure composed of nonredundant subsets of GO terms, where the distance between GO terms was measured based on the semantic similarity [19].

2.12. Pathway Analyses. A pathway enrichment analysis based on Fisher's exact test was performed using DAVID [17]. As with GO analysis, significantly enriched pathways were identified from an input list of genes and statistically adjusted using FDR. For a more systematic pathway analysis, we conducted a Signaling Pathway Impact Analysis (SPIA) [20], which identifies pathways relevant to the experimental conditions using a list of differentially expressed genes and their expression ratios combined with signaling pathway topology. By randomly bootstrapping the pathway topology $(n=3,000)$, two statistical measurements, $P_{\mathrm{NDE}}$ and $P_{\mathrm{PERT}}$, were calculated, which measure the overrepresentation of input genes in a pathway and the abnormal perturbation of a specific pathway, respectively. The global $P$ value $\left(P_{\mathrm{G}}\right)$ calculated from $P_{\mathrm{NDE}}$ and $P_{\mathrm{PERT}}$ was used as the selection criteria for significant pathways.

The pathway analysis methods outlined above primarily focused on the identification of enriched pathways using differentially expressed genes. We then measured dosedependent changes in pathway activity by calculating a linear combination of the logarithmic value of the expression of all of the genes in each pathway, with a weight of 1 . When the genes acted as repressors, the weight was multiplied by -1 . The measured activities were normalized and hierarchically clustered. The statistical significance for each pathway was estimated using the random permutation-based method $(n=$ $1,000)[21]$ in which the FDR was determined by comparing the activity values with randomly permutated values. Only pathways with an FDR below 0.05 were included in the clustering analysis. The pathway information used in the present study was obtained from the Kyoto Encyclopedia of Genes and Genomes (KEGG, http://www.genome.jp/kegg/) database.

2.13. Pathway Similarity Matrix. Pathway similarity was measured based on the number of common genes between pathways. Briefly, a matrix of the number of common genes in distinct pathways was constructed and the relative similarity was measured using the Jaccard algorithm [22] in which the fraction of common genes between two pathways was used to calculate similarity. Therefore, the absence of a common gene in two pathways was not considered in measuring similarity. Finally, the relative similarity matrix was hierarchically clustered, and the pathway activity values obtained by linear combinations of the expression ratio, as described above, were merged into a similarity matrix.

\section{Results}

3.1. Cytotoxic Effects of the EEDS on Human Lung Cancer Cells. To determine the cytotoxic effects of the EEDS, A549 human lung cancer cells were exposed to increasing concentrations of the EEDS or the vehicle control for indicated time periods. 


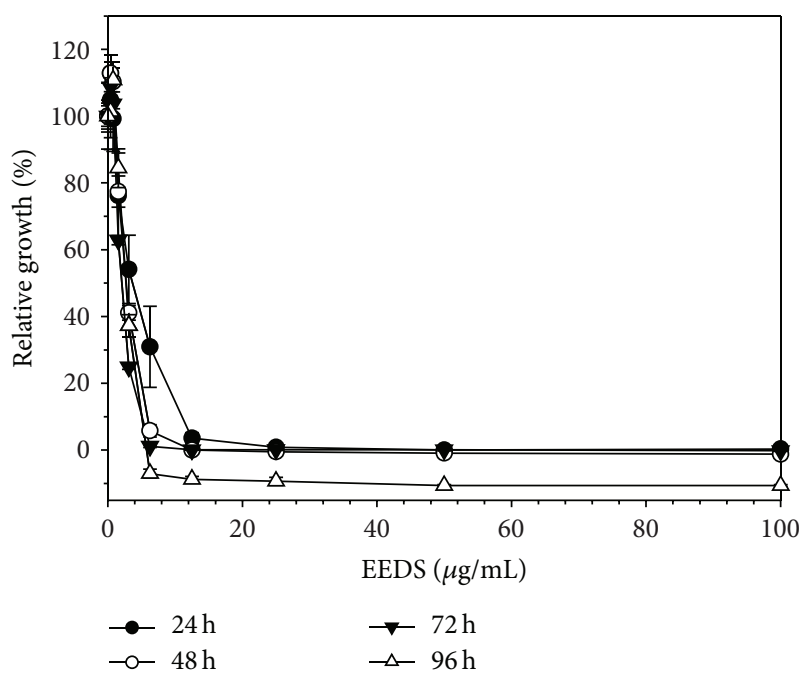

(a)

$\operatorname{EEDS}(\mu \mathrm{g} / \mathrm{mL})$
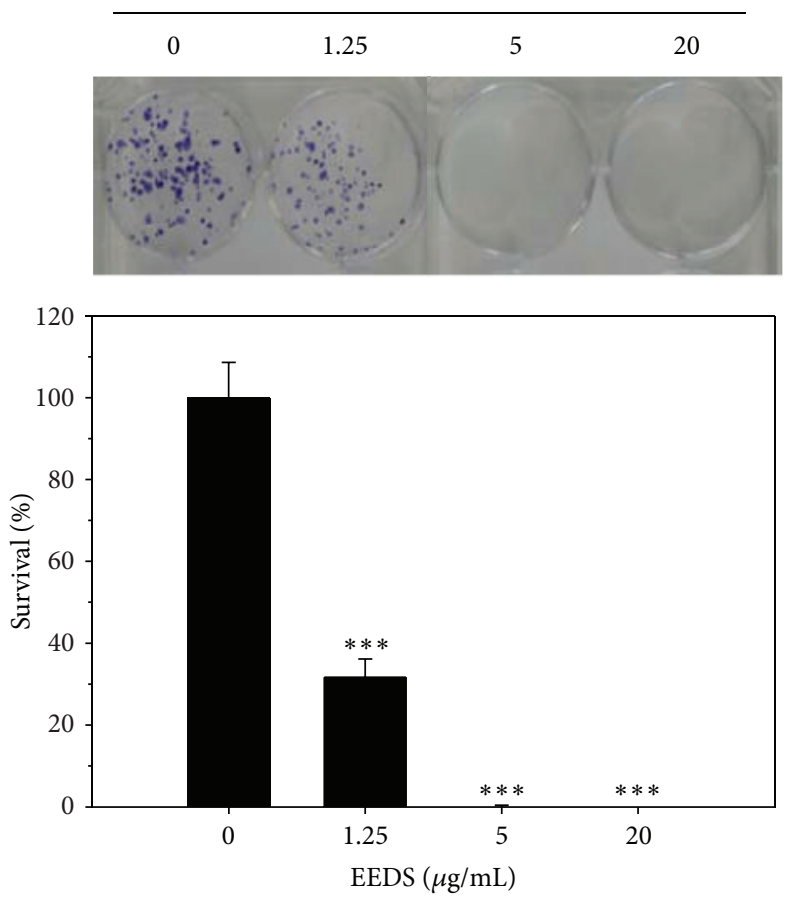

(b)
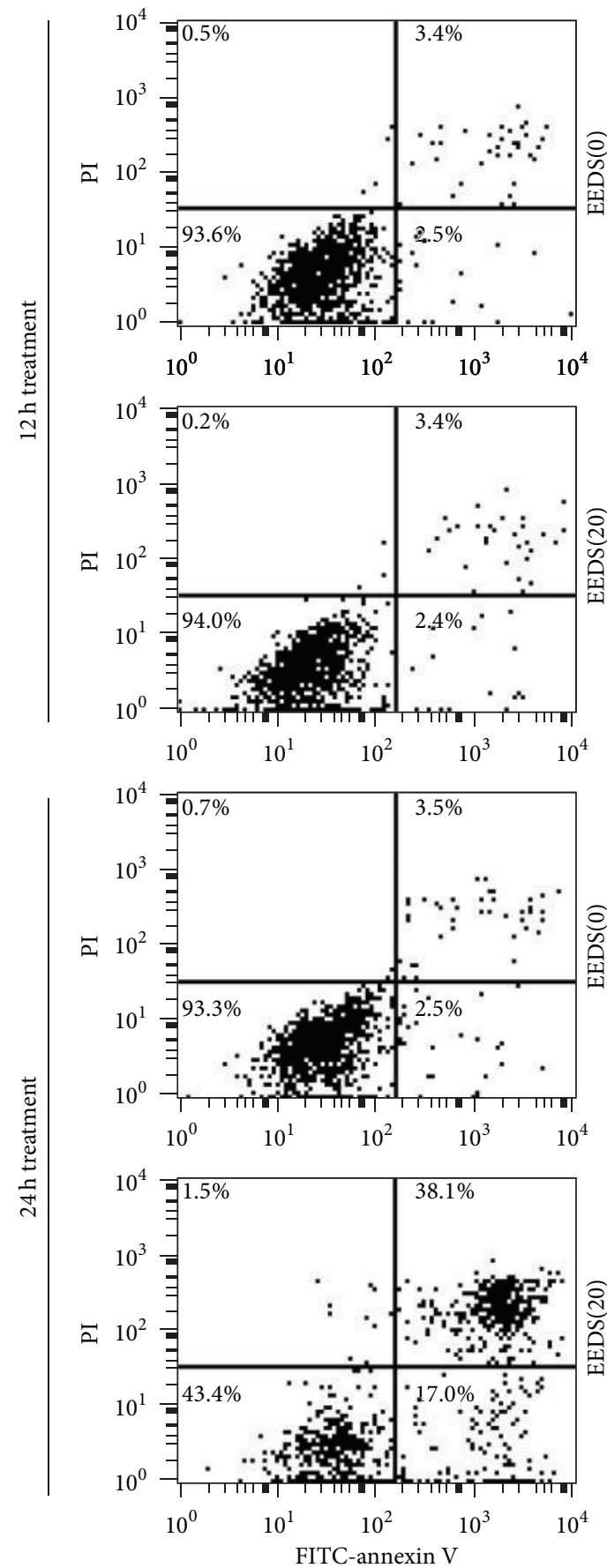

(c)

FIGURE 1: Cytotoxic effect of EEDS on A549 human lung cancer cells. (a) A549 was exposed to increasing concentrations $(0-100 \mu \mathrm{g} / \mathrm{mL})$ of EEDS for various time periods (24-96 h). Cell viability was determined based on mitochondrial enzyme activity as described in the Materials and Methods section. The relative cell growth at each drug dose was calculated by comparison with the vehicle control $(0 \mu \mathrm{g} / \mathrm{mL}$ of EEDS, $0.5 \%$ DMSO) treatment. (b) A549 cells were exposed to increasing concentrations $(0-20 \mu \mathrm{g} / \mathrm{mL})$ of EEDS for $72 \mathrm{~h}$ and then incubated for an additional 10 days in the absence of EEDS (upper panel). Colonies stained with crystal violet were counted and expressed as relative survival (\%) compared to the vehicle control (0.5\% DMSO) treatment (lower panel). All data are presented as the mean \pm S.D. of triplicate experiments. The differences between the vehicle control and treated groups were determined using Student's $t$-test. ${ }^{* * *} P<0.001$. (c) Representative FITCAnnexin V/PI scatter plots for A549 cells following the EEDS treatment. A549 cells were exposed to EEDS $(0 \mathrm{or} 20 \mu \mathrm{g} / \mathrm{mL})$ for $12 \mathrm{~h}$ or $24 \mathrm{~h}$, and then subjected to FACS analysis of FITC-Annexin V and PI. 
TABLE 1: IC $_{50}$ of EEDS in human lung cancer cell lines.

\begin{tabular}{|c|c|c|c|c|c|}
\hline \multirow{2}{*}{ Cell lines } & \multirow{2}{*}{ NCI60 panel } & \multirow{2}{*}{ Disease } & \multirow{2}{*}{ Drug expose (h) } & \multicolumn{2}{|c|}{$\mathrm{IC}_{50}$} \\
\hline & & & & $\operatorname{EEDS}(\mu \mathrm{g} / \mathrm{mL})$ & $\operatorname{Dox}(\mu \mathrm{M})^{\mathrm{a}}$ \\
\hline \multirow{4}{*}{ A549 } & \multirow{4}{*}{ Yes } & \multirow{4}{*}{ Lung cancer } & 24 & $3.72 \pm 1.12$ & n.d. \\
\hline & & & 48 & $2.81 \pm 0.19$ & $0.04 \pm 0.00$ \\
\hline & & & 72 & $2.15 \pm 0.01$ & n.d. \\
\hline & & & 96 & $2.59 \pm 0.16$ & n.d. \\
\hline NCI-H23 & Yes & Lung cancer & 48 & $6.60 \pm 0.18$ & $0.22 \pm 0.04$ \\
\hline NCI-H226 & Yes & Lung cancer & 48 & $13.18 \pm 0.77$ & $0.33 \pm 0.04$ \\
\hline NCI-H460 & Yes & Lung cancer & 48 & $8.08 \pm 0.01$ & $0.31 \pm 0.04$ \\
\hline IMR-90 & - & Normal lung & 48 & $10.54 \pm 0.79$ & $0.40 \pm 0.04$ \\
\hline
\end{tabular}

${ }^{a}$ Dox, doxorubicin, was included as a reference anticancer drug [12].

As shown in Figure 1(a), the EEDS efficiently inhibited A549 cell growth in a dose-dependent manner. Three other human lung cancer cell lines (NCI-H23, NCI-H226, and NCI-H460) and the IMR-90 normal human lung fibroblast cell line were also exposed to increasing concentrations of the EEDS for $48 \mathrm{~h}$. The half maximal inhibitory concentrations (IC50s) of the EEDS against different cells lines were calculated and are summarized in Table 1. Among the tested cell lines, A549 $(2.81 \pm 0.19 \mu \mathrm{g} / \mathrm{mL})$ cells were the most sensitive, while NCI$\mathrm{H} 226(13.18 \pm 0.77 \mu \mathrm{g} / \mathrm{mL})$ and IMR-90 $(10.54 \pm 0.79 \mu \mathrm{g} / \mathrm{mL})$ were relatively resistant to EEDS. We also performed colony forming assays to determine whether the EEDS could affect the tumorigenic ability of A549 cells. The results indicated that treatment with 5 and $20 \mu \mathrm{g} / \mathrm{mL}$ of the EEDS for $72 \mathrm{~h}$ completely inhibited colony formation from single cells, whereas cells treated with the vehicle control $(0 \mu \mathrm{g} / \mathrm{mL}$ of EEDS, $0.5 \%$ DMSO) were able to form colonies (Figure 1(b), upper panel). Relatively lower colony numbers were observed in A549 cells treated with a low concentration of the EEDS $(1.25 \mu \mathrm{g} / \mathrm{mL})$ (Figure $1(\mathrm{~b})$, lower panel). In order to elucidate how EEDS can inhibit cell proliferation, we assessed apoptotic cell death of A549 cells following EEDS treatment. Relative to the vehicle control, the percentage of A549 cells undergoing early (Annexin $\mathrm{V}$ positive and PI negative) and late (Annexin V positive and PI positive) apoptotic cell death was increased after $24 \mathrm{~h}$ treatment of $20 \mu \mathrm{g} / \mathrm{mL}$ of EEDS. Taken together, these data suggest that the EEDS can inhibit cell proliferation and reduce the tumorigenicity of A549 cells through induction of apoptotic cell death. We selected A549 cells that had been treated with the EEDS for further analyses of gene expression profiling.

3.2. Gene Expression Profiles. The overall pattern of gene expression in A549 cells after EEDS treatment is shown in Figure 2(a). Two subgroups of genes that were upregulated and downregulated in dose-dependent manners were identified. To obtain more quantitative analysis, we applied a dose-dependency analysis to the gene expression values. In accordance with the clustering profile of the genes, two significantly different patterns (Down- and Up-patterns) were observed (FDR < 0.001). The Down-pattern consisted of 1,680 genes that were downregulated in a dose-dependent manner, and the Up-pattern consisted of 1,673 genes that were upregulated in a dose-dependent manner. Expression plots of the two patterns are presented in Figure 2(b). The top 20 genes that displayed the greatest amount of variations in each pattern are listed in Table 2. In addition, the expression ratios for all of the genes included in Figure 2(a) are indicated in Supplementary Table 2. The results of the expression chip analysis were validated using semi-qPCR reactions of 10 selected genes displaying UP- and Downpatterns (Figure 2(c)).

3.3. Prognostic Implications. We investigated whether dosedependent alteration in gene expression is implicated in clinical outcomes of lung cancer. First, using publically available lung cancer data (GSE4573), we identified survivalrelated genes (log-rank $P$ value $<0.05)$. We then examined the relationship between these prognostic genes and UPor Down-pattern genes. As shown in Table 3, 48 Downpattern and 50 Up-pattern genes were among the survivalrelated genes identified from lung cancer patients. Among the 48 Down-pattern genes, 32 Down-pattern genes displayed high-hazard ratios $(>1)$, and 16 genes displayed low-hazard ratios $(<1)$. However, there were a greater number of Uppattern genes with low-hazard ratios (26 genes) than those with high-hazard ratios (24 genes). Although this reciprocal distribution of genes was marginally significant $(P$ value of 0.069 in Fisher Exact test), considering that genes with highhazard ratios were downregulated, while genes with lowhazard ratios were upregulated, we hypothesized that EEDS treatment may enhance antitumorigenic effects.

3.4. GO Analysis. To identify the biological function of the two patterns, a GO analysis was performed. The Downpattern gene set was enriched with metabolic GO terms, including cofactor biosynthesis, heterocycle biosynthesis, and nitrogen compound biosynthesis. In contrast, signalingrelated GO terms, including transcription regulation, protein kinase regulation, and apoptosis regulation, were enriched in the Up-pattern gene set. The top 10 statistically significant categories of GO terms (FDR $<0.01$ ) are shown in Table 4 (for the full list of enriched GO terms, please see Supplementary Table 4).

3.5. Profiling of GO Terms. The simple GO analysis considered only genes included in the Down- or Up-pattern 
TABLE 2: Top 20 list of genes mostly down-regulated or up-regulated by EEDS.

\begin{tabular}{|c|c|c|c|c|c|c|c|}
\hline \multicolumn{4}{|c|}{ Down-pattern } & \multicolumn{4}{|c|}{ Up-pattern } \\
\hline \multirow{2}{*}{ Symbol } & \multicolumn{3}{|c|}{$\operatorname{EEDS}(\mu \mathrm{g} / \mathrm{mL})$} & \multirow{2}{*}{ Symbol } & \multicolumn{3}{|c|}{$\operatorname{EEDS}(\mu \mathrm{g} / \mathrm{mL})$} \\
\hline & 1.25 & 5 & 20 & & 1.25 & 5 & 20 \\
\hline CSRP2BP & $-1.35^{*}$ & -3.05 & -6.16 & HBEGF & 0.86 & 1.58 & 4.44 \\
\hline SCARA5 & -0.28 & -2.58 & -5.71 & C7orf53 & 2.86 & 2.73 & 4.46 \\
\hline HOXB13 & -0.66 & -2.42 & -5.06 & SERPINE1 & 1.54 & 3.50 & 4.59 \\
\hline FOXS1 & 0.12 & -2.40 & -4.92 & CD274 & 2.59 & 3.23 & 4.60 \\
\hline SNORA12 & -2.24 & -4.44 & -4.91 & NOG & 1.16 & 3.91 & 4.67 \\
\hline RIMBP3 & -2.00 & -2.74 & -4.89 & PER1 & 2.22 & 1.52 & 4.68 \\
\hline ST6GAL1 & -1.82 & -2.20 & -4.88 & ARC & 0.78 & 1.69 & 4.79 \\
\hline DACT2 & -2.47 & -4.08 & -4.87 & GADD45B & 1.23 & 1.95 & 4.82 \\
\hline TMEM37 & -1.42 & -4.37 & -4.85 & LTB & 1.37 & 4.06 & 4.85 \\
\hline EEF2K & -1.55 & -2.50 & -4.84 & FOS & 1.25 & 0.52 & 4.87 \\
\hline GPRIN2 & -2.57 & -3.41 & -4.79 & DDIT3 & 1.66 & 2.78 & 4.90 \\
\hline SALL2 & -2.08 & -4.10 & -4.76 & C3orf52 & 1.04 & 2.26 & 4.96 \\
\hline CBR3 & -1.56 & -3.26 & -4.57 & MAFF & 1.62 & 2.70 & 5.07 \\
\hline FANCF & -1.30 & -1.88 & -4.56 & IL8 & 2.94 & 4.52 & 5.55 \\
\hline CCR7 & -0.58 & -1.72 & -4.47 & PPP1R15A & 1.42 & 2.61 & 5.59 \\
\hline C5orf58 & -0.90 & -1.72 & -4.38 & LOC387763 & 3.52 & 4.33 & 5.67 \\
\hline VAV3 & -0.91 & -3.48 & -4.36 & ATF3 & 1.02 & 2.49 & 5.69 \\
\hline C14orf93 & -0.64 & -2.00 & -4.31 & EGR2 & 0.11 & 0.72 & 6.46 \\
\hline NTHL1 & -0.57 & -2.05 & -4.31 & FOSB & 1.36 & 3.75 & 7.85 \\
\hline VASH1 & -1.31 & -3.69 & -4.28 & EGR1 & 1.44 & 4.10 & 10.02 \\
\hline
\end{tabular}

${ }^{*}$ Fold induction represents $\log _{2}$ expression ratio of gene compared with that of control.

gene sets. To identify dose-dependent changes in GO terms, all differentially expressed genes were considered in the analysis. As shown in Figure 3(a), most GO terms were altered by treatment with the highest EEDS concentration tested $(20 \mu \mathrm{g} / \mathrm{mL}$; FDR $<0.01)$. Consistent with the results of the simple GO analysis, the major functions altered were associated with apoptosis and signaling processes.

The enriched GO categories included redundant terms, however, and it was therefore necessary to remove duplicate terms. We used the REVIGO program to obtain nonredundant GO terms (FDR < 0.01) that were altered by EEDS treatment and to measure the functional relationship of these terms in the network structure. Figure 3(b) shows that signaling-related GO terms, including apoptosis, the MAPK cascade, protein kinase regulation, and phosphorylation regulation, were connected with each other as a cluster, suggesting an interrelationship of biological processes after EEDS treatment in A549 cells.

3.6. Pathway Analyses. In addition to the GO analysis, we also examined the functional changes induced by EEDS treatment by performing pathway analyses. Enriched pathways (FDR < 0.01 ) identified from the Down- and the Up-pattern gene sets are listed in Table 5. Although only the base excision repair pathway (KEGG 03410) and pentose phosphate pathway (KEGG 00030) were significantly enriched in the Downpattern gene set, signaling-related pathways, including the MAPK pathway (KEGG 4010), the apoptosis pathway (KEGG
4210), the p53 pathway (KEGG 4155), and the TGF-beta pathway, were enriched in the Up-pattern gene set. The list and positions of the Up- or Down-pattern genes in the pathways are depicted in Supplementary Figure 2. For more systematic analyses of the pathways, we conducted SPIA pathway analyses, which calculate a $P$ value for a pathway based on random perturbations to the pathway network topology. In the Down-pattern gene set (Figure 4(a)), only the sulfur relay system pathway (KEGG 4211) was significant $\left(P_{\mathrm{G}}<0.01\right)$, whereas in the Up-pattern gene set (Figure 4(b)), the MAPK pathway (KEGG 4010), the apoptosis pathway (KEGG 4210), the p53 pathway (KEGG 4155), and the TGFbeta pathway were significant $\left(P_{\mathrm{G}}<0.01\right)$. These results are consistent with those of the simple pathway enrichment analysis (Table 5). We also obtained similarly enriched pathways using the combined Down- and Up-pattern gene sets (Figure 4(c)).

3.7. Pathway Activity Analyses. Sequential changes in pathway activities based on the EEDS treatment dose were measured using a linear combination of the expression values of all genes in each pathway. Two major subclusters of pathways were grouped based on the statistically significant (FDR < 0.01) similarity of pathway activities (Figure 5(a)). Subcluster 1, which is composed of pathways with activities that decreased in a dose-dependent manner, is associated with several metabolism-related pathways. Sub-cluster 2, which is composed of pathways with activities that increased in a 
TABLE 3: Clinical association of Down- or Up-pattern genes regulated by the EEDS with survival from lung cancer.

\begin{tabular}{|c|c|c|c|c|c|c|c|}
\hline \multicolumn{4}{|c|}{ Down-pattern } & \multicolumn{4}{|c|}{ Up-pattern } \\
\hline Symbol & Fold induction* & $P$ value ${ }^{* *}$ & Hazard ratio** & Symbol & Fold induction & $P$ value & Hazard ratio \\
\hline HMBS & -2.06 & 0.00225 & 1.41 & YPEL5 & 1.99 & 0.000577 & 0.62 \\
\hline GMPPA & -2.80 & 0.00283 & 1.46 & MNT & 1.56 & 0.00344 & 1.42 \\
\hline CD79A & -1.27 & 0.00431 & 0.72 & GGA3 & 1.11 & 0.00421 & 1.40 \\
\hline HSPA12A & -1.18 & 0.00759 & 1.43 & MGC29506 & 1.00 & 0.00471 & 0.72 \\
\hline RUNX3 & -1.19 & 0.00818 & 0.71 & CHD7 & 1.63 & 0.00763 & 1.49 \\
\hline RBKS & -1.17 & 0.0123 & 1.47 & ERCC6 & 1.52 & 0.00887 & 1.37 \\
\hline LRRC20 & -2.88 & 0.0132 & 1.47 & SSBP2 & 1.61 & 0.0122 & 0.72 \\
\hline RNF144A & -1.37 & 0.0136 & 0.75 & NUFIP1 & 1.41 & 0.0129 & 1.33 \\
\hline C11orf60 & -1.30 & 0.0148 & 1.36 & CLK3 & 1.04 & 0.0159 & 1.37 \\
\hline BCS1L & -1.12 & 0.0149 & 1.32 & PPP1R13L & 2.74 & 0.0160 & 1.35 \\
\hline CHST12 & -2.05 & 0.0155 & 0.75 & TNFSF9 & 1.92 & 0.0162 & 1.34 \\
\hline CRYBB2P1 & -1.09 & 0.0172 & 0.75 & DDX52 & 1.80 & 0.0163 & 1.36 \\
\hline APITD1 & -2.35 & 0.0176 & 1.35 & FAM108B1 & 1.33 & 0.0192 & 0.73 \\
\hline AP1G2 & -1.62 & 0.0177 & 1.39 & ZMYM5 & 1.23 & 0.0203 & 1.32 \\
\hline ALDH3B1 & -1.77 & 0.0185 & 1.36 & PTPRH & 1.89 & 0.0217 & 1.34 \\
\hline AUTS2 & -1.89 & 0.0210 & 0.74 & BTG2 & 3.33 & 0.0231 & 0.74 \\
\hline NIPSNAP1 & -1.64 & 0.0229 & 1.29 & ARHGEF15 & 1.52 & 0.0233 & 1.3 \\
\hline LCMT1 & -1.69 & 0.0240 & 1.29 & SPATA2L & 1.68 & 0.0244 & 1.30 \\
\hline HERC6 & -1.18 & 0.0250 & 0.75 & PRKRIP1 & 1.56 & 0.0245 & 0.76 \\
\hline DYRK4 & -1.21 & 0.0261 & 0.77 & KIFC1 & 1.47 & 0.0247 & 1.32 \\
\hline TPD52L1 & -1.30 & 0.0262 & 1.35 & RUNX1 & 2.83 & 0.0247 & 1.32 \\
\hline VPS33B & -1.05 & 0.0274 & 1.32 & GOLGA8A & 2.02 & 0.0249 & 0.72 \\
\hline DHRS1 & -1.83 & 0.0280 & 1.28 & ABL1 & 1.15 & 0.0254 & 0.76 \\
\hline WDR61 & -1.26 & 0.0292 & 1.32 & CRABP2 & 2.34 & 0.0258 & 0.75 \\
\hline PYGL & -1.62 & 0.0314 & 1.31 & DDIT3 & 4.91 & 0.0260 & 1.34 \\
\hline MKS1 & -1.08 & 0.0316 & 1.29 & IP6K2 & 2.19 & 0.0273 & 0.76 \\
\hline VASH1 & -4.29 & 0.0336 & 0.77 & LOC729806 & 1.2 & 0.0275 & 1.36 \\
\hline MRPL46 & -1.52 & 0.0338 & 1.29 & ELL & 2.63 & 0.0284 & 0.79 \\
\hline APBA2 & -2.56 & 0.0340 & 0.75 & TRAF4 & 1.00 & 0.0294 & 1.39 \\
\hline LARGE & -2.96 & 0.0341 & 0.76 & SEMA7A & 1.16 & 0.0297 & 1.31 \\
\hline IMP3 & -1.40 & 0.0354 & 1.28 & PRNP & 1.51 & 0.0303 & 0.75 \\
\hline AAAS & -1.55 & 0.0354 & 0.78 & GADD45A & 4.03 & 0.0314 & 0.76 \\
\hline TGIF1 & -1.41 & 0.0359 & 1.26 & GLIPR1 & 1.94 & 0.0330 & 0.77 \\
\hline C11orf80 & -1.03 & 0.0363 & 1.32 & PAPOLG & 1.93 & 0.0331 & 0.75 \\
\hline AGAP11 & -1.51 & 0.0387 & 1.33 & ZNF484 & 2.81 & 0.0336 & 1.36 \\
\hline CCR7 & -4.47 & 0.0390 & 0.78 & RAP2C & 1.46 & 0.0336 & 0.76 \\
\hline RRBP1 & -1.05 & 0.0395 & 0.77 & MED1 & 1.25 & 0.0389 & 1.24 \\
\hline $\mathrm{CDC} 123$ & -1.09 & 0.0422 & 1.29 & HERPUD1 & 1.01 & 0.0392 & 0.79 \\
\hline REEP4 & -1.48 & 0.0433 & 1.28 & DDR2 & 1.11 & 0.0404 & 0.79 \\
\hline PFKFB1 & -1.40 & 0.0433 & 0.78 & PLK4 & 1.59 & 0.0417 & 1.30 \\
\hline ZNF839 & -2.28 & 0.0440 & 1.3 & CDC42SE1 & 2.29 & 0.0417 & 0.76 \\
\hline CCDC53 & -1.88 & 0.0460 & 0.80 & $\mathrm{AOC} 2$ & 1.09 & 0.0430 & 1.29 \\
\hline RDX & -1.22 & 0.0466 & 1.29 & RUNX2 & 1.80 & 0.0438 & 1.29 \\
\hline EIF2B3 & -2.05 & 0.0470 & 1.27 & SFRS12IP1 & 1.81 & 0.0451 & 0.76 \\
\hline PAAF1 & -1.81 & 0.0471 & 1.35 & INPP1 & 1.40 & 0.0456 & 0.76 \\
\hline PITPNC1 & -1.39 & 0.0483 & 1.29 & MCAM & 1.57 & 0.0458 & 0.79 \\
\hline ATIC & -1.31 & 0.0492 & 1.27 & LTB & 4.85 & 0.0470 & 0.77 \\
\hline \multirow[t]{3}{*}{ DDX28 } & -1.63 & 0.0498 & 1.28 & ZCCHC10 & 1.13 & 0.0480 & 0.79 \\
\hline & & & & SP2 & 1.59 & 0.0489 & 0.79 \\
\hline & & & & PTHLH & 2.81 & 0.0499 & 0.79 \\
\hline
\end{tabular}

* Fold induction represents $\log _{2}$ expression ratio of gene at treatment with $20 \mu \mathrm{g} / \mathrm{mL}$ of EEDS.

${ }^{* *}$ Log-rank $P$ value and hazard ratio were measured in Cox-proportional hazard regression model performed in public lung cancer data [16]. 


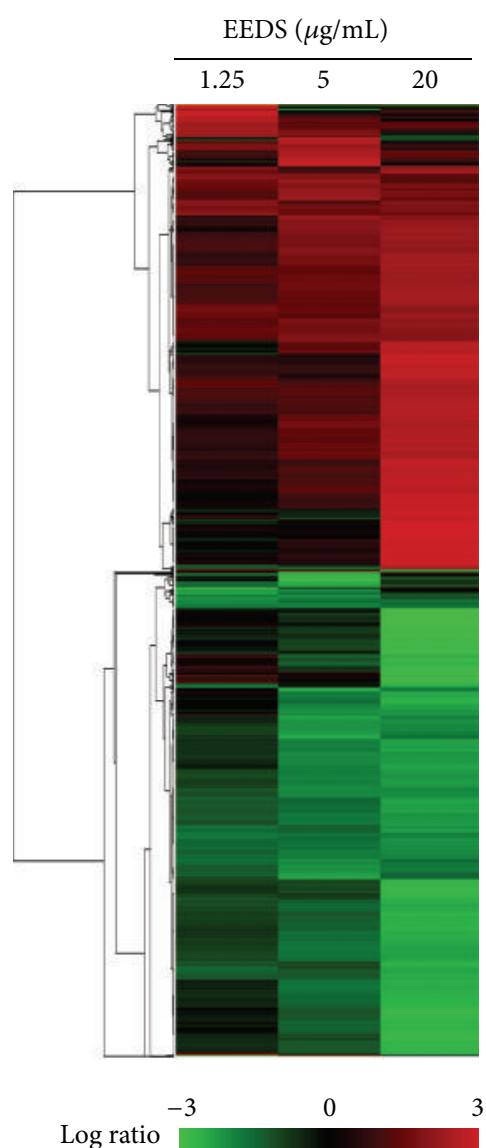

(a)
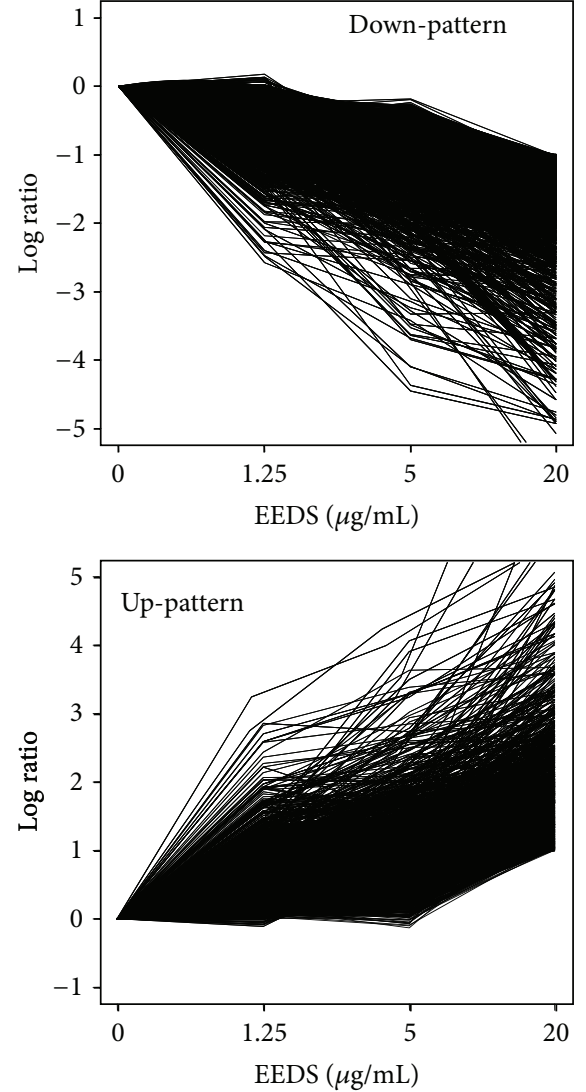

(b)

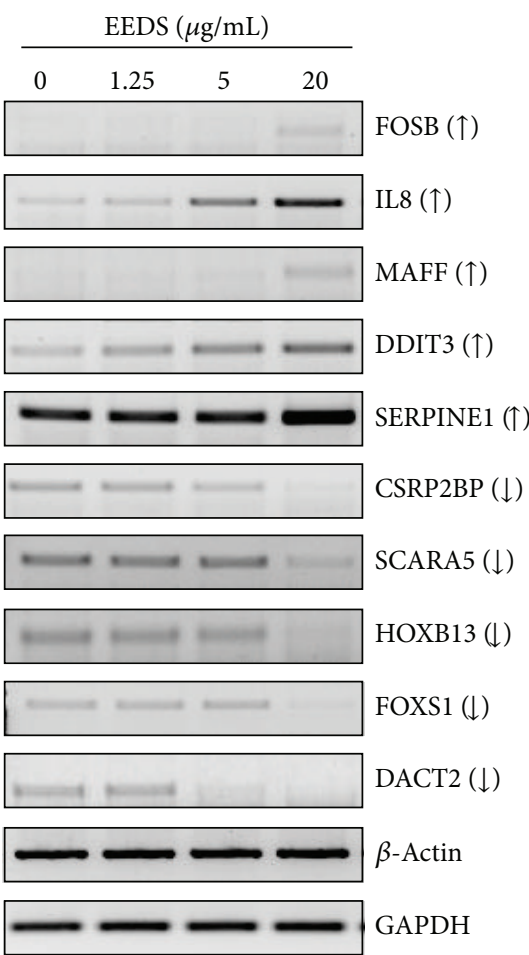

(c)

FIGURE 2: Dose-dependent gene expression by EEDS treatment in A549 cells. (a) Approximately 5,400 differentially expressed genes with a fold ratio greater than 2 or less than 0.5 (for up- and down-regulation, resp.) compared to the vehicle control group in at least one sample were clustered hierarchically. Columns and rows represent individual samples and genes, respectively. The expression ratio color scale ranges from red (high) to green (low), as indicated by the scale bar. Genes exhibiting statistically significant dose-dependent alterations were identified via the STEM program (FDR < 0.001). (b) The Down-pattern is composed of 1,680 genes and the Up-pattern is composed of 1,673 genes. (c) Data acquired from expression chip analysis was confirmed by semi-qPCR. Ten selected genes displaying Up- $(\uparrow)$ and Down- $(\downarrow)$ patterns were amplified using gene specific primers. $\beta$-actin and GAPDH were used as loading controls.

dose-dependent manner, is associated with signaling-related pathways as well as immune- and disease-related pathways. To identify the relationship between the statistically significant pathways, we constructed a similarity matrix of pathways based on component genes and pathway activities. As shown in Figure 5(b), subgroups of pathways were clustered based on the similarities of their component genes. Among them, one large subgroup was composed of signaling- and immunerelated pathways. The full list of pathways is presented in Supplementary Figure 3. The pathway activities, depicted on a diagonal line or "Activity" on the right panel, indicate that pathways clustered in a subgroup have common levels of activity. For example, the signaling- and immune-related pathways that clustered in the same subgroup show similarly increased activities, suggesting that the interconnection of these diverse pathways may be involved in the response mechanism of A549 cells to EEDS treatment.

\section{Discussion}

Our previous study demonstrated that the EEDS is cytotoxic to human cancer cell lines and that a cardiac glycoside (helveticoside) is an active cytotoxic constituent of the EEDS [6]. In accordance with our previous work, the EEDS significantly inhibited cell growth and tumorigenicity in A549 human non-small cell lung carcinoma cells through induction of apoptotic cell death (Figure 1). Although the major cytotoxic constituent (helveticoside) of the EEDS was previously identified, the cellular mechanism underlying the therapeutic effects of the EEDS was not. One of the main limitations in elucidating the therapeutic mechanism of whole extracts is the complexity of the biological processes affected by the diverse components of extracts. Therefore, it is difficult to reveal the biological pathways associated with herbal drug treatment using a conventional approach based on the 


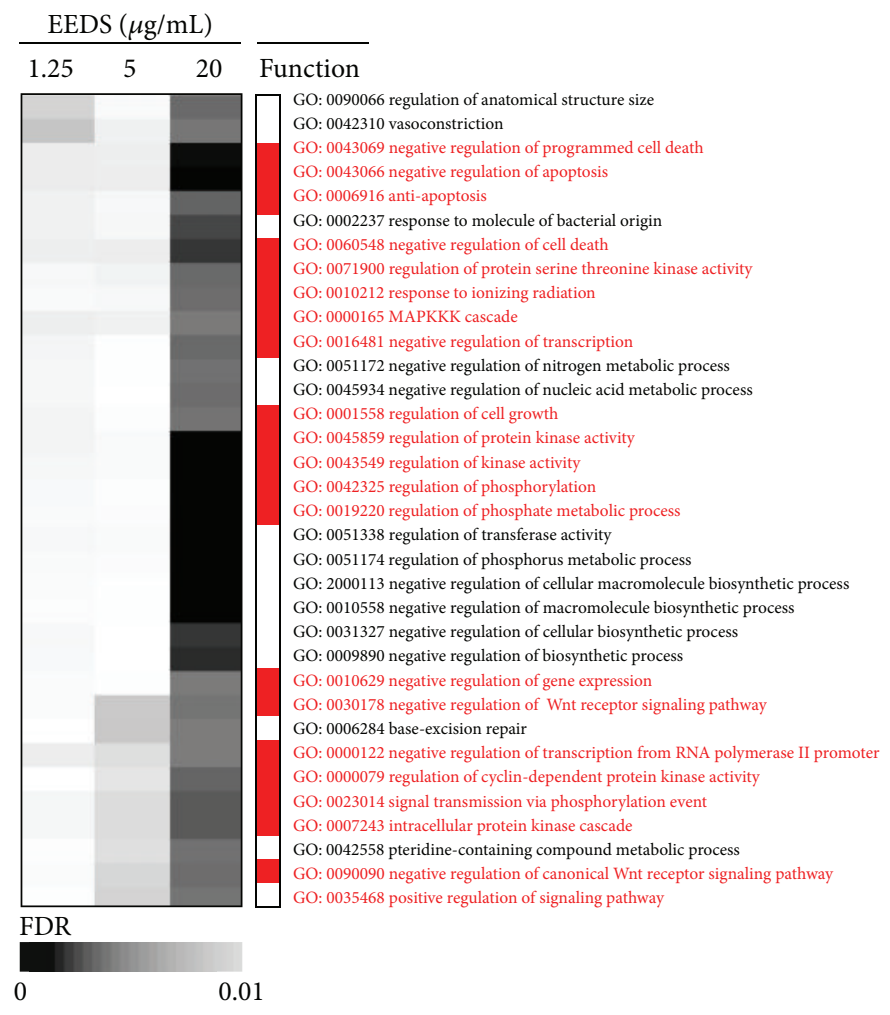

Signal-related pathways
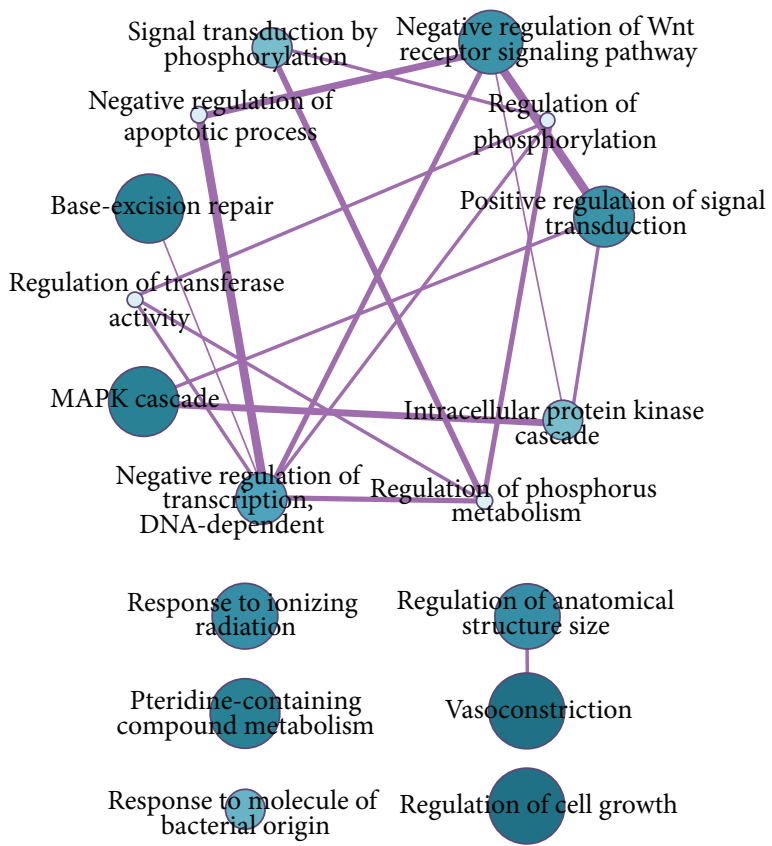

(b)

FIGURE 3: Distribution of GO terms altered by EEDS treatment in A549 cells. (a) GO terms associated with differentially expressed genes that had a fold ratio greater than 2 or less than 0.5 (for up- and down-regulation, resp.) were analyzed at each dose of EEDS using the HighThroughput GoMiner tool. Columns represent individual samples, and rows represents statistically significant GO terms (FDR < 0.01). The positions of signaling-related pathways are colored red. Statistical significance is represented by a gray color gradient, as indicated by the scale bar. (b) A network composed of all nonredundant statistically significant GO terms (FDR $<0.01)$ after EEDS treatment $(20 \mu \mathrm{g} / \mathrm{mL})$ was constructed using the REVIGO program. The size and color density of each GO term are proportional to its statistical significance, and edge thickness represents the relatedness between two nodes.

analysis of a handful of genes. In general, administration of herbal drugs induces or represses a large number of genes across the whole genome. In the present study, roughly 5,400 genes (approximately 25\% of all genes) were found to be differentially expressed following EEDS treatment, as shown in Figure 2(a). Given that treatment with $20 \mu \mathrm{g} / \mathrm{mL}$ of the EEDS significant inhibited cell growth, we also investigated whether the genes regulated by the EEDS are involved in cell growth signaling functions.

Among the differentially expressed genes, two statistically significant patterns of gene expression were observed (Figure 2(b)). The functional segregation of the two gene expression patterns was then validated in two manners. First, we investigated the clinical associations of the two patterns by comparing the gene sets with survival-related genes that were obtained from a public lung cancer dataset. Interestingly, there was a tendency for high-risk genes in lung cancer to be more heavily distributed in the Downpattern gene set (32 versus 16), while low-risk genes were more heavily distributed in the Up-pattern gene set (26 versus 24$)$, with marginal statistical significance ( $P$ value of
0.069). Although the number of genes that were common to the Up- or Down-pattern gene sets and survival-related genes was small, our results suggest that the EEDS could be effective in prolonging survival by inhibiting high-risk genes and activating low-risk genes. Second, differences in functional involvement between the Down-pattern and Uppattern gene sets were measured using GO and pathway analyses. For example, genes grouped in the Down-pattern gene set, which exhibited dose-dependent decrease in expression, were involved in heterogeneous functions, such as metabolic processes or the base excision repair pathway, while genes in the Up-pattern gene set were predominately associated with cell growth signaling functions (Figure 4 and Table 5). Previous reports indicated that energy metabolism involving the pentose phosphate pathway can regulate lung cancer cells [23] and polymorphisms in the base excision repair pathway are related to lung cancer risk [24] and can modulate the effectiveness of chemotherapy in lung cancer patients [25]. Cell growth-related pathways including the MAPK pathway (KEGG 4010), the apoptosis pathway (KEGG 4210), the p53 pathway (KEGG 4155), and the TGF-beta pathway 
TABLE 4: Top 10 GO terms associated with Up- and Down-patterns by EEDS treatment.

\begin{tabular}{clc}
\hline GO ID & GO terms & $P$ value $^{*}$ \\
\hline Down-pattern & & $9.26 E-08$ \\
GO:0051188 & Cofactor biosynthetic process & $1.79 E-07$ \\
GO:0051186 & Cofactor metabolic process & $8.03 E-07$ \\
GO:0018130 & Heterocycle biosynthetic process & $6.15 E-06$ \\
GO:0044271 & Nitrogen compound biosynthetic process & $1.65 E-05$ \\
GO:0006399 & tRNA metabolic process & $2.57 E-04$ \\
Up-pattern & & $4.21 E-26$ \\
GO:0045449 & Regulation of transcription & $3.75 E-21$ \\
GO:0006350 & Transcription & $3.11 E-14$ \\
GO:0006355 & Regulation of transcription, DNA-dependent & $3.78 E-14$ \\
GO:0051252 & Regulation of RNA metabolic process & $2.05 E-13$ \\
GO:0006357 & Regulation of transcription from RNA polymerase II promoter & $2.04 E-03$ \\
GO:0042325 & Regulation of phosphorylation & $2.03 E-11$ \\
GO:0051173 & Positive regulation of nitrogen compound metabolic process & $2.39 E-11$ \\
GO:0045859 & Regulation of protein kinase activity & $3.92 E-11$ \\
GO:0019220 & Regulation of phosphate metabolic process & $3.49 E-11$ \\
GO:0051174 & Regulation of phosphorus metabolic process & $1.51 E-10$ \\
\hline
\end{tabular}

* $P$ values were calculated using Fischer's test.

${ }^{* *}$ FDR corrections were calculated using the Benjamini-Hochberg procedure in DAVID program [17].

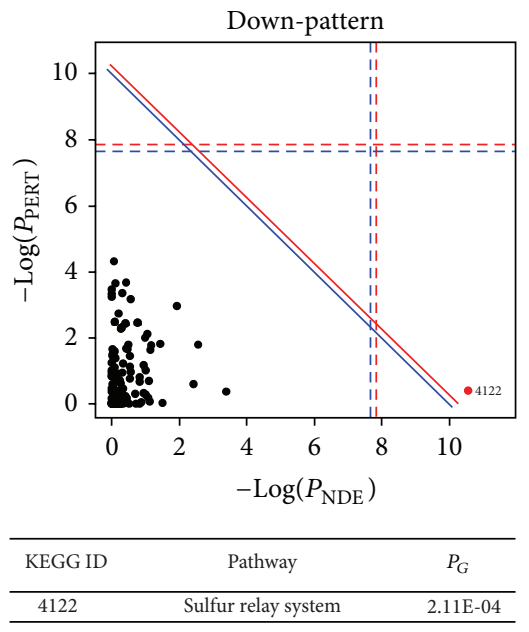

(a)

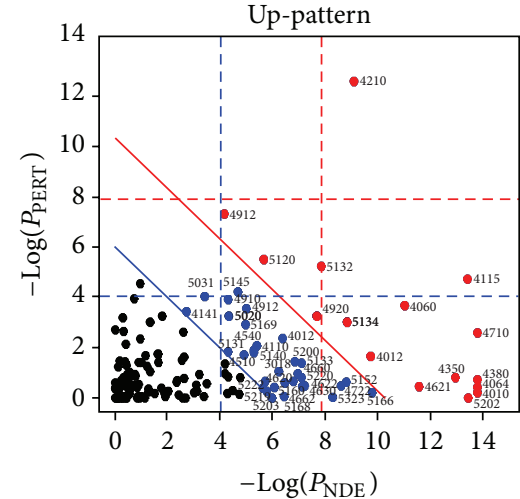

\begin{tabular}{ccc}
\hline $\begin{array}{c}\text { KEGG } \\
\text { ID }\end{array}$ & Pathway & $P_{G}$ \\
\hline 4210 & Apoptosis & $8.34 E-09$ \\
4010 & MAPK signaling pathway & $474 E-08$ \\
4115 & p53 signaling pathway & $2.39 E-07$ \\
4710 & Circadian rhythm & $3.37 E-07$ \\
4380 & Osteoclast differentiation & $1.9 E-06$ \\
4064 & NF- $\kappa$ B signaling pathway & $4.74 E-06$ \\
4060 & Cytokine-cytokine receptor interaction & $6.34 E-06$ \\
4350 & TGF- $\beta$ signaling pathway & $1.54 E-05$ \\
5202 & Transcriptional misregulation in cancer $2.08 E-05$ \\
5132 & Salmonella infection & $2.92 E-05$ \\
4621 & NOD-like receptor signaling pathway & $7.62 E-05$ \\
5134 & Legionellosis & $9.11 E-05$ \\
4912 & GnRH signaling pathway & $1.28 E-04$ \\
4012 & ErbB signaling pathway & $1.38 E-04$ \\
5120 & Epithelial cell signaling in Helicobacter & $1.67 E-04$ \\
4920 & pylori infection & Adipocytokine signaling pathway \\
\hline
\end{tabular}

(b)

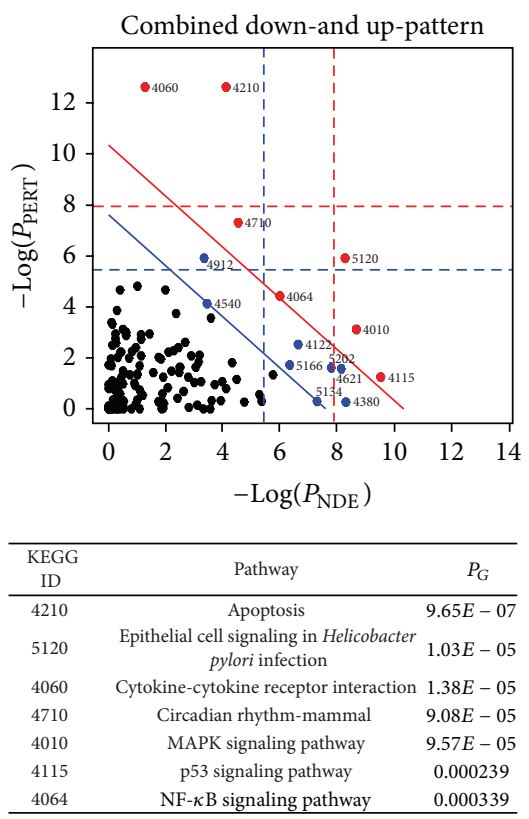

(c)

FIgURE 4: Pathways altered by EEDS treatment in A549 cells. Statistically significant pathways in (a) the Down-pattern, (b) the Up-pattern, and (c) the combined Down- and Up-patterns were analyzed by implementing the SPIA program. The horizontal or vertical axis represents the overrepresentation of a pathway $\left(P_{\mathrm{NDE}}\right)$ or the perturbation of a pathway $\left(P_{\mathrm{PERT}}\right)$, respectively. Red or blue dotted lines represent the Bonferroni- or FDR- corrected thresholds of significance (1\%), respectively, for each axis value. Red and blue circles are significant pathways after Bonferroni and FDR correction $(1 \%)$ of the global $P$ values $\left(P_{\mathrm{G}}\right)$, respectively. A list of pathways consisting of red circles (Bonferroni corrected $\left.P_{\mathrm{G}}<0.01\right)$ is shown below. 


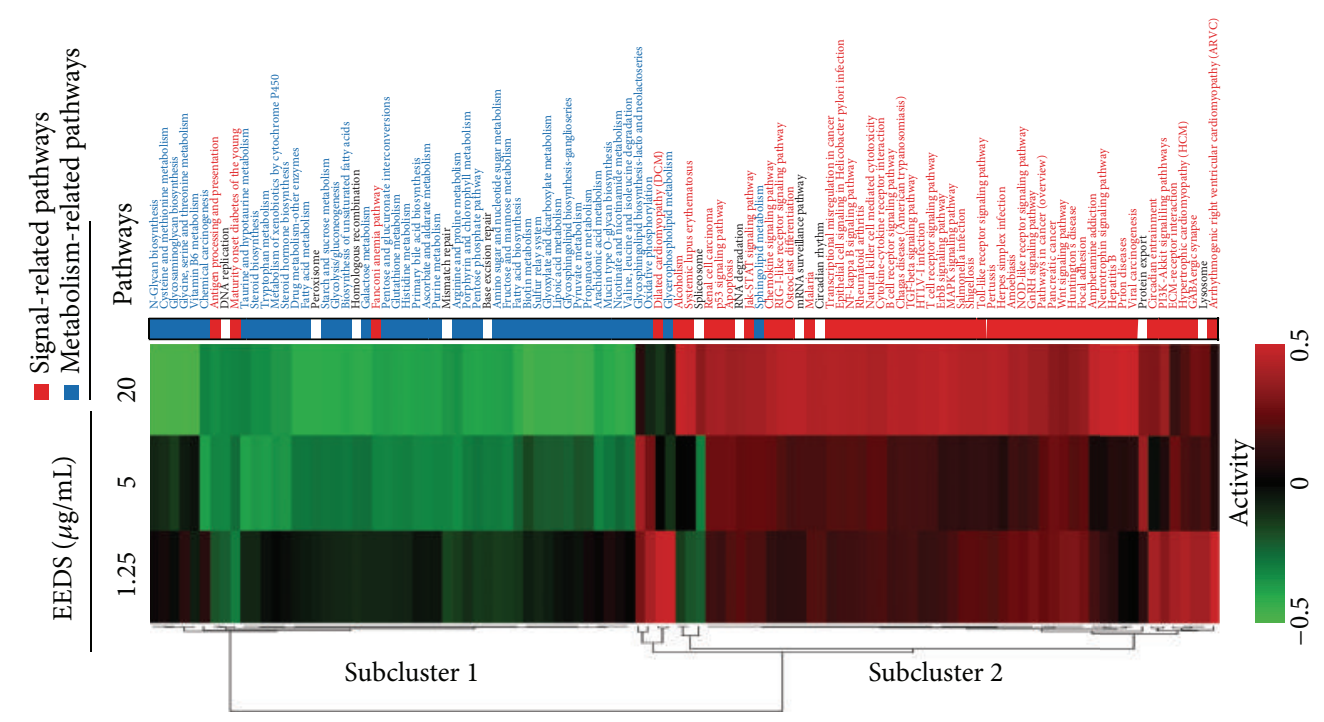

(a)

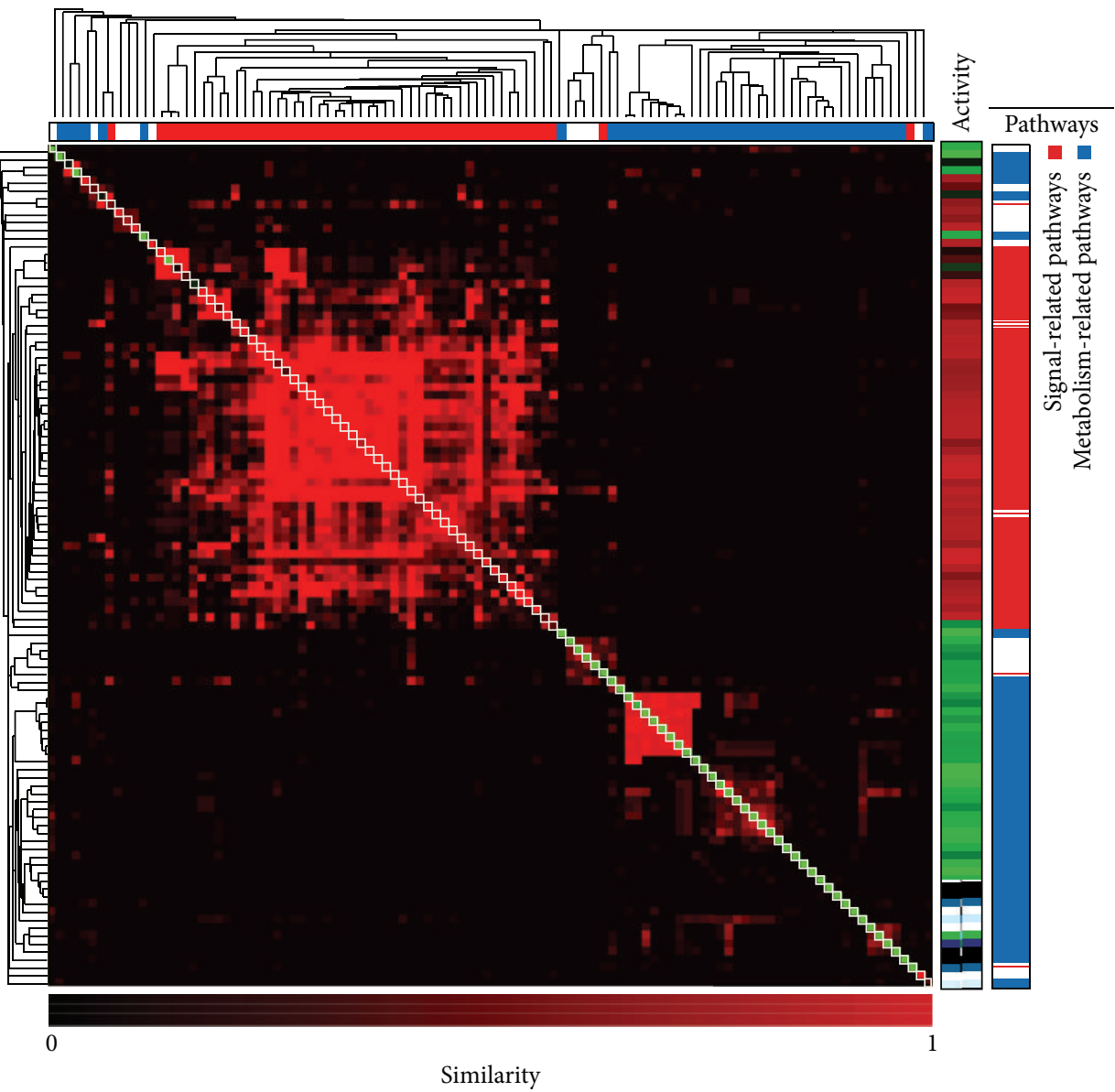

(b)

FIGURE 5: Pathway activities altered by EEDS treatment in A549 cells. (a) The dose-dependent change in pathway activities was measured and clustered hierarchically. Columns represent individual samples, and rows represent activities of pathways. The pathway activity color scale ranges from red (high) to green (low), as indicated by scale bars with arbitrary units. The pathway positions are colored green to indicate metabolic pathways and red to indicate signaling-related pathways. (b) After measurement of the similarity between pathways, the matrix of similarity was clustered hierarchically and merged with activities of pathways. The level of similarity is represented in red with a scale bar. The color in the box of a diagonal line or "Activity" on the right panel represents the activity of the pathway. The positions of signaling-related pathways are colored red and the metabolism-related pathways are colored blue. 
TABLE 5: Pathways associated with Up- and Down-patterns by EEDS treatment.

\begin{tabular}{|c|c|c|c|}
\hline KEGG ID & Pathway & $P$ value ${ }^{*}$ & $\mathrm{FDR}^{* *}$ \\
\hline \multicolumn{4}{|c|}{ Down-pattern } \\
\hline 03410 & Base excision repair & $3.90 E-04$ & $6.66 E-03$ \\
\hline 00030 & Pentose phosphate pathway & $1.47 E-04$ & $1.22 E-03$ \\
\hline \multicolumn{4}{|l|}{ Up-pattern } \\
\hline 04010 & MAPK signaling pathway & $1.16 E-07$ & $1.83 E-05$ \\
\hline 04210 & Apoptosis & $4.99 E-06$ & $3.94 E-04$ \\
\hline 04115 & p53 signaling pathway & $2.46 E-05$ & $1.29 E-03$ \\
\hline 04350 & TGF-beta signaling pathway & $5.36 E-05$ & $2.11 E-03$ \\
\hline 04060 & $\begin{array}{l}\text { Cytokine-cytokine receptor } \\
\text { interaction }\end{array}$ & $1.14 E-04$ & $3.60 E-03$ \\
\hline 04710 & Circadian rhythm & $3.20 E-04$ & $8.38 E-03$ \\
\hline 04621 & $\begin{array}{l}\text { NOD-like receptor signaling } \\
\text { pathway }\end{array}$ & $3.39 E-04$ & $7.61 E-03$ \\
\hline
\end{tabular}

${ }^{*} P$ values were calculated using Fischer's test.

${ }^{* *}$ FDR corrections were calculated using the Benjamini-Hochberg procedure [17].

were also significantly enriched in the Up-pattern gene set $\left(P_{\mathrm{G}}<0.01\right)$, raising the possibility that the EEDS stimulates functionally related biological pathways (Figure 4(b)). The MAPK pathway has been widely reported to be involved in the growth and invasion of lung cancer, and this pathway has been used for the development of anti-lung cancer drugs [2628]. Activation of the apoptosis and p53 pathways is also one of the main targets of anti-lung cancer drugs, including herbal extracts [29-31]. Other functionally related pathways identified in the Up-pattern gene set included immune systemor infectious disease-related pathways, including cytokinecytokine receptor interaction (KEGG 04060), NOD-like receptor signaling pathway (KEGG 04621), Helicobacter pylori infection (KEGG 05120), and Salmonella infection (KEGG 05132). Interestingly, immune-system regulation has been reported to improve lung cancer patient outcomes [32, 33]. Similar functional involvement was also observed when we performed a pathway analysis using all of the differentially expressed genes contained in the Down- or the Up-pattern gene sets (Figure 4(c)).

In addition to the Down- and Up-pattern gene sets, we used the expression values from all genes to measure changes in pathway activities. Intriguingly, the results of this analysis clearly demonstrated that metabolism-related pathways and signaling-related pathways were regulated in dose-dependent and reciprocal manners (Figure 5(a)). The activities of a group of metabolism-related pathways were significantly diminished, whereas a group of signaling pathways, including apoptosis and immune-related pathways, were significantly activated in a dose-dependent manner. The regulatory pattern observed in our system is in agreement with the postulated anti-carcinogenic effects of the EEDS, as previously proposed by many reports in which the inhibition of metabolic pathways and the activation of apoptosis or immune-related pathways were the main targets of lung cancer drug development $[23,26,29,31,32]$. The observation that the activities of diverse signaling and metabolic pathways appeared in separate clusters implies the existence of a common reciprocal regulatory mechanism. Therefore, we also measured the relationships between pathways based on the similarities of pathway component genes and integrated pathway activities. The results of this analysis indicate that signaling pathways with increased activity are grouped in a large cluster, suggesting that diverse signaling pathways are similarly affected by EEDS treatment (Figure 5(b)). In contrast, the results show that metabolic pathways with decreased activity are grouped in a small cluster. Our results show that the signaling and metabolic pathways were interconnected through the complex network structure.

Despite these data, the growth inhibitory effect of the EEDS is difficult to explain. To fully elucidate the molecular mechanism underlying the activity of the EEDS, the biological implication of the reciprocal regulation of two biologically distinct groups of pathways must be determined and the exact relationships between the diverse pathways should be verified in more detail. Moreover, further rigorous studies should be done to determine whether the observed reciprocal regulation of biological functions is a general mechanism of herbal extracts. Nonetheless, our present results provide evidence in support of the importance of using whole genome approaches to elucidate pharmaceutical mechanisms.

\section{Conclusion}

In conclusion, the results of the present study indicate that EEDS treatment induces dose-dependent responses in A549 human non-small cell lung carcinoma cells that involve the up-regulation of a large group of genes associated with cell growth-related signaling pathways and the downregulation of genes associated with metabolic function. This reciprocal regulatory mechanism may provide clues to further our understanding of the mechanism driving growth inhibition in human cancer cells treated with the EEDS, especially in A549 human lung cancer cells.

\section{Conflict of Interests}

The authors have declared that there are no competing interests associated with this paper.

\section{Acknowledgments}

The authors wish to thank Ms. Deok Rim Heo for her technical assistance in acquiring FACS data. This research was supported by a Grant from the Korea Institute of Oriental Medicine (KIOM, K13061).

\section{References}

[1] G. Ghavami, M. R. Kazemali, and S. Sardari, "Informatics of drug synergism in naturally occurring anticancer agents," Recent Patents on Anti-Cancer Drug Discovery, vol. 6, no. 1, pp. 26-44, 2011.

[2] J.-S. Chia, J.-L. Du, W.-B. Hsu, A. Sun, C.-P. Chiang, and W.B. Wang, "Inhibition of metastasis, angiogenesis, and tumor 
growth by Chinese herbal cocktail Tien-Hsien Liquid," $B M C$ Cancer, vol. 10, article 175, 2010.

[3] H. Wagner and G. Ulrich-Merzenich, "Synergy research: approaching a new generation of phytopharmaceuticals," Phytomedicine, vol. 16, no. 2-3, pp. 97-110, 2009.

[4] S. Faivre, S. Djelloul, and E. Raymond, "New paradigms in anticancer therapy: targeting multiple signaling pathways with kinase inhibitors," Seminars in Oncology, vol. 33, no. 4, pp. 407420, 2006.

[5] S. Sathornsumetee, "Therapeutic strategies to target multiple kinases in glioblastoma," Anti-Cancer Agents in Medicinal Chemistry, vol. 11, no. 8, pp. 700-711, 2011.

[6] Y. J. Lee, N. S. Kim, H. Kim et al., "Cytotoxic and antiinflammatory constituents from the seeds of Descurainia sophia," Archives of Pharmacal Ressearch, vol. 36, no. 5, pp. 536541, 2013.

[7] Y. Q. Chen, R. Z. Li, and Y. W. Wang, "Identification of cardiac glycosides from the seeds of Descurainia sophia L. Webb," Acta Pharmaceutica Sinica, vol. 16, no. 1, pp. 62-64, 1981.

[8] K. Sun, X. Li, J.-M. Liu, J.-H. Wang, W. Li, and Y. Sha, "A novel sulphur glycoside from the seeds of Descurainia sophia (L.)," Journal of Asian Natural Products Research, vol. 7, no. 6, pp. 853856, 2005.

[9] K. Sun, X. Li, W. Li, J.-M. Liu, J.-H. Wang, and Y. Sha, "A new nor-lignan from the seeds of Descurainia sophia," Natural Product Research, vol. 20, no. 6, pp. 519-522, 2006.

[10] K. Sun, X. Li, W. Li, J. Wang, J. Liu, and Y. Sha, "Two new lactones and one new aryl-8-oxa-bicyclo[3,2,1]oct-3-en2-one from Descurainia sophia," Chemical and Pharmaceutical Bulletin, vol. 52, no. 12, pp. 1483-1486, 2004.

[11] T. M. Ehrman, D. J. Barlow, and P. J. Hylands, "In silico search for multi-target anti-inflammatories in Chinese herbs and formulas," Bioorganic and Medicinal Chemistry, vol. 18, no. 6, pp. 2204-2218, 2010.

[12] Y. J. Lee, J. Kim, J. M. Yi et al., "Anti-proliferative neolignans from Saururus chinensis against human cancer cell lines," Biological \& Pharmaceutical Bulletin, vol. 35, no. 8, pp. 13611366, 2012.

[13] A. Schroeder, O. Mueller, S. Stocker et al., "The RIN: an RNA integrity number for assigning integrity values to RNA measurements," BMC Molecular Biology, vol. 7, article 3, 2006.

[14] B. M. Bolstad, R. A. Irizarry, M. Åstrand, and T. P. Speed, "A comparison of normalization methods for high density oligonucleotide array data based on variance and bias," Bioinformatics, vol. 19, no. 2, pp. 185-193, 2003.

[15] J. Ernst and Z. Bar-Joseph, "STEM: a tool for the analysis of short time series gene expression data," BMC Bioinformatics, vol. 7, article 191, 2006.

[16] M. Raponi, Y. Zhang, J. Yu et al., "Gene expression signatures for predicting prognosis of squamous cell and adenocarcinomas of the lung," Cancer Research, vol. 66, no. 15, pp. 7466-7472, 2006.

[17] G. Dennis Jr., B. T. Sherman, D. A. Hosack et al., "DAVID: database for annotation, visualization, and integrated discovery," Genome Biology, vol. 4, no. 5, article P3, 2003.

[18] B. R. Zeeberg, H. Qin, S. Narasimhan et al., "High-throughput GoMiner, an "industrial-strength" integrative gene ontology tool for interpretation of multiple-microarray experiments, with application to studies of Common Variable Immune Deficiency (CVID)," BMC Bioinformatics, vol. 6, article 168, 2005.
[19] F. Supek, M. Bošnjak, N. Škunca, and T. Šmuc, "Revigo summarizes and visualizes long lists of gene ontology terms," PLoS ONE, vol. 6, no. 7, Article ID e21800, 2011.

[20] A. L. Tarca, S. Draghici, P. Khatri et al., "A novel signaling pathway impact analysis," Bioinformatics, vol. 25, no. 1, pp. 7582, 2009.

[21] S. Y. Lee, K. H. Song, I. Koo et al., "Comparison of pathways associated with hepatitis $\mathrm{B}$ - and C-infected hepatocellular carcinoma using pathway-based class discrimination method," Genomics, vol. 99, no. 6, pp. 347-354, 2012.

[22] S. Veerla and M. Höglund, "Analysis of promoter regions of co-expressed genes identified by microarray analysis," $B M C$ Bioinformatics, vol. 7, article 384, 2006.

[23] V. P. Sukhatme and B. Chan, "Glycolytic cancer cells lacking 6phosphogluconate dehydrogenase metabolize glucose to induce senescence," FEBS Letters, vol. 586, no. 16, pp. 2389-2395, 2012.

[24] W. Zhao, L. Hu, J. Xu et al., "Polymorphisms in the base excision repair pathway modulate prognosis of platinum-based chemotherapy in advanced non-small cell lung cancer," Cancer Chemotherpy and Pharmacology, vol. 71, no. 5, pp. 1287-1295, 2013.

[25] C. Kiyohara, K. Takayama, and Y. Nakanishi, "Association of genetic polymorphisms in the base excision repair pathway with lung cancer risk: a meta-analysis," Lung Cancer, vol. 54, no. 3, pp. 267-283, 2006.

[26] Z. Lu, L. Ding, H. Hong, J. Hoggard, Q. Lu, and Y.-H. Chen, "Claudin-7 inhibits human lung cancer cell migration and invasion through ERK/MAPK signaling pathway," Experimental Cell Research, vol. 317, no. 13, pp. 1935-1946, 2011.

[27] H. Ji, Z. Wang, S. A. Perera et al., "Mutations in BRAF and KRAS converge on activation of the mitogen-activated protein kinase pathway in lung cancer mouse models," Cancer Research, vol. 67, no. 10, pp. 4933-4939, 2007.

[28] A. A. Adjei, "The role of mitogen-activated ERK-kinase inhibitors in lung cancer therapy," Clinical Lung Cancer, vol. 7, no. 3, pp. 221-223, 2005.

[29] S. Mohan, S. I. Abdelwahab, S. C. Cheah et al., "Apoptosis effect of girinimbine isolated from Murraya koenigii on lung cancer cells in vitro," Evididence-Based Complementary and Alternative Medicine, vol. 2013, Article ID 689865, 12 pages, 2013.

[30] Y. F. Ho, S. A. Karsani, W. K. Yong, and S. N. Abd Malek, "Induction of apoptosis and cell cycle blockade by helichrysetin in a549 human lung adenocarcinoma cells," Evididence-Based Complementary and Alternative Medicine, vol. 2013, Article ID 857257, 10 pages, 2013.

[31] T. Choedon, D. Dolma, and V. Kumar, "Pro-apoptotic and anticancer properties of thapring-a Tibetan herbal formulation," Journal of Ethnopharmacology, vol. 137, no. 1, pp. 320-326, 2011.

[32] F. A. Shepherd, J.-Y. Douillard, and G. R. Blumenschein Jr., "Immunotherapy for non-small cell lung cancer: novel approaches to improve patient outcome," Journal of Thoracic Oncology, vol. 6, no. 10, pp. 1763-1773, 2011.

[33] G. Mazzoccoli, M. Grilli, S. Carughi et al., "Immune system alterations in lung cancer patients," International Journal of Immunopathology and Pharmacology, vol. 16, no. 2, pp. 167-174, 2003. 


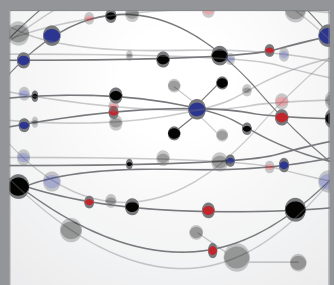

The Scientific World Journal
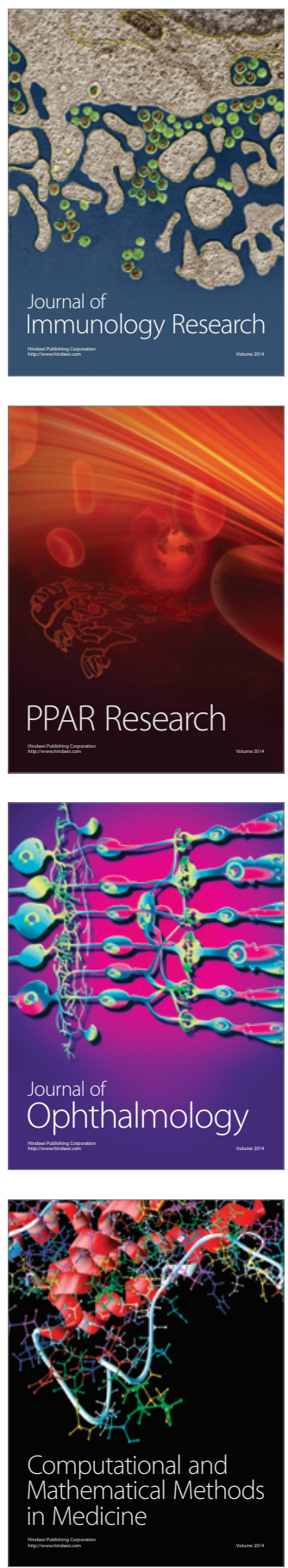

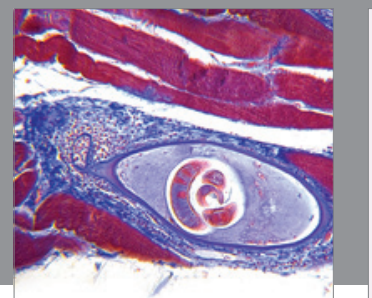

Gastroenterology

Research and Practice
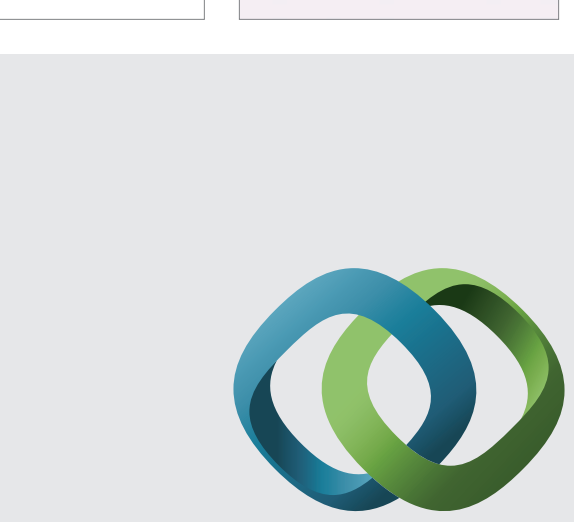

\section{Hindawi}

Submit your manuscripts at

http://www.hindawi.com
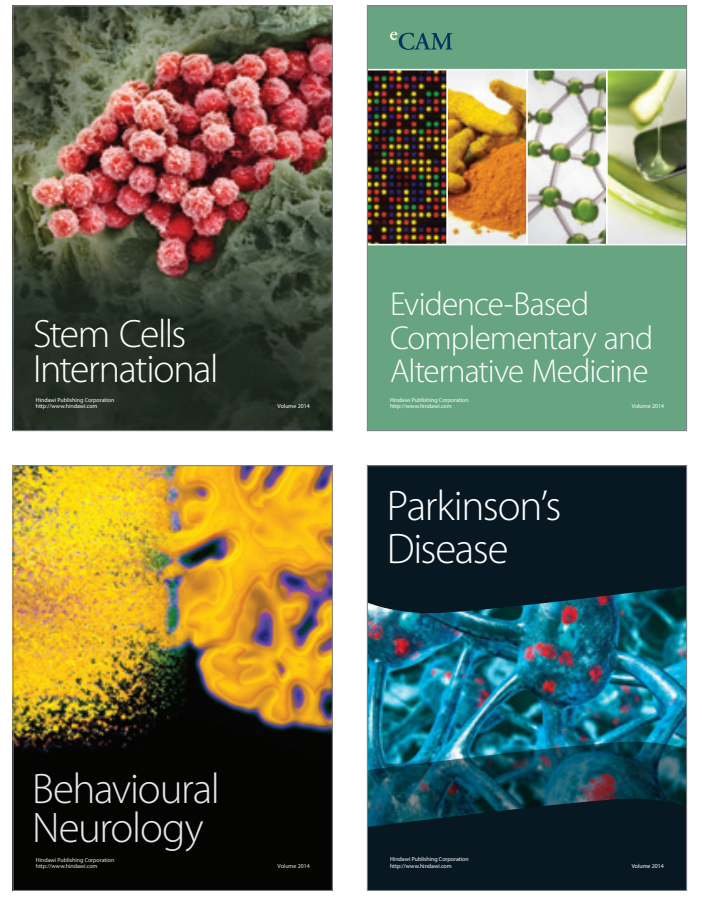
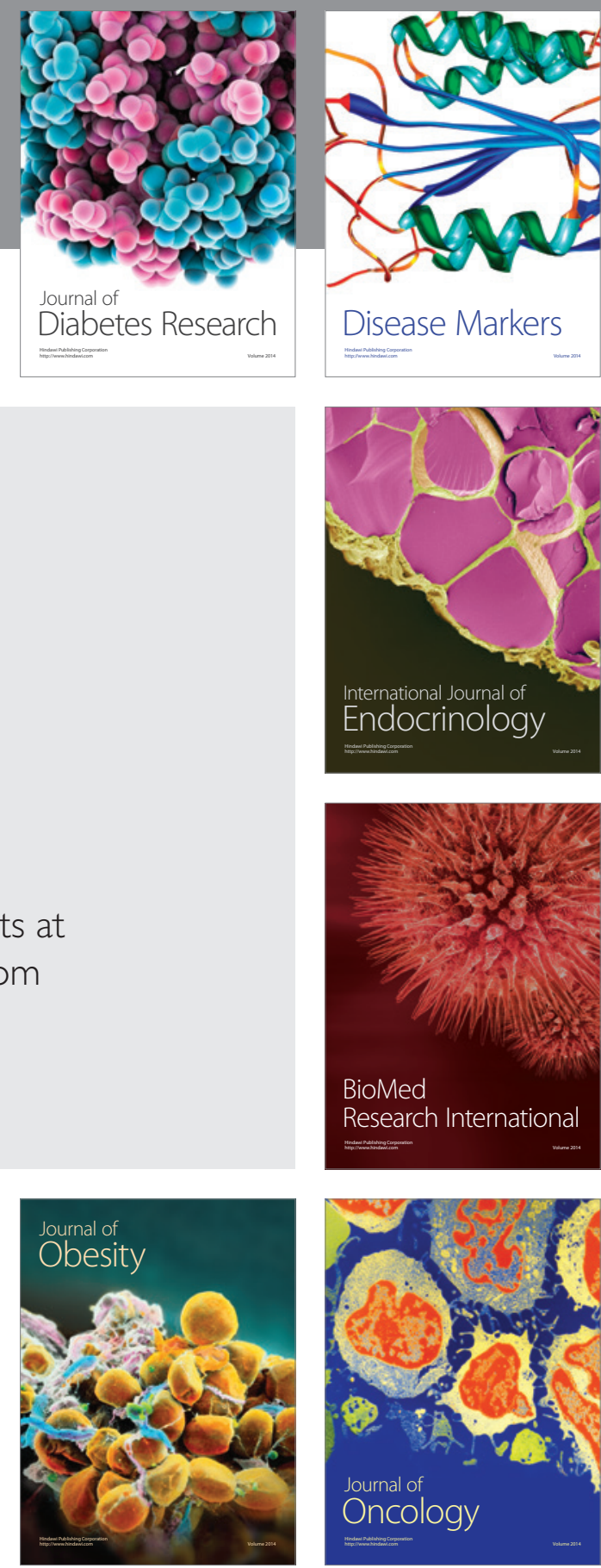

Disease Markers
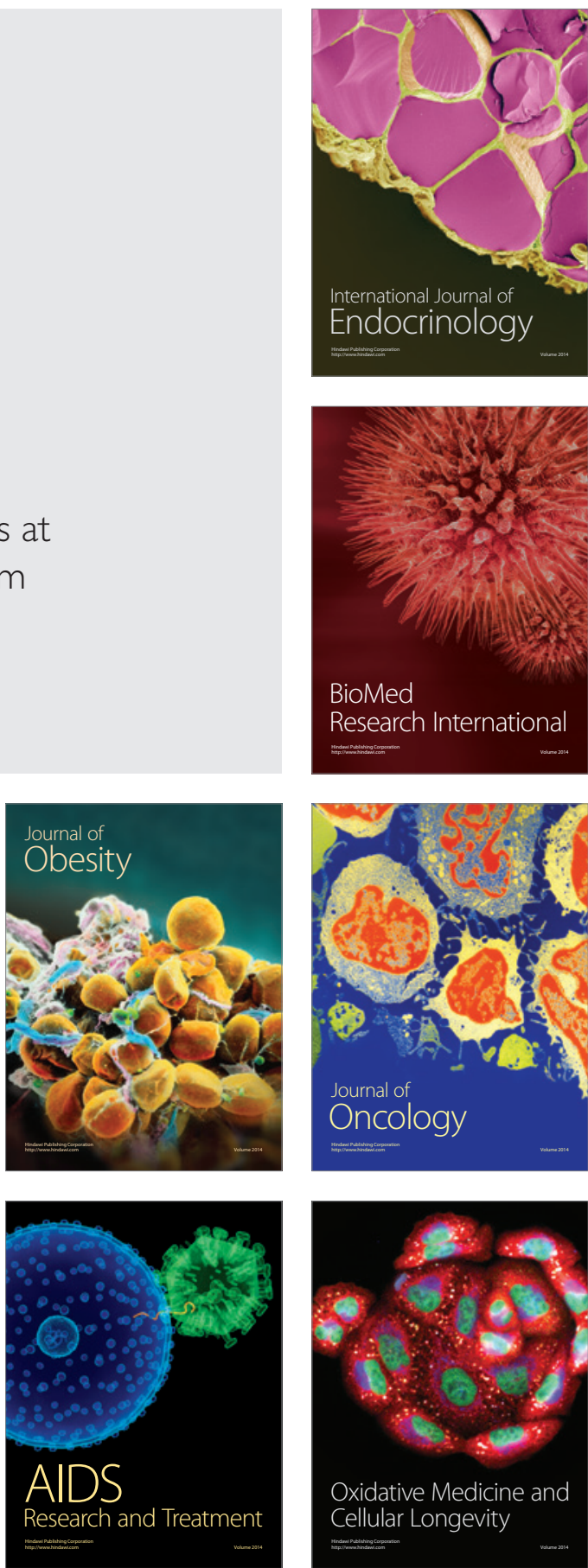